\title{
Application of a Real-Fluid Turbomachinery Analysis to Rocket Turbopump Geometries
}

\author{
Daniel J. Domey* \\ NASA Marshall Space Flight Center \\ Applied Fluid Dynamics Analysis Group \\ MSFC, AL 35812 \\ Douglas L. Sondak ${ }^{+}$ \\ Boston University \\ Office of Information Technology \\ Boston, MA 02215 \\ Bogdan Marcu \\ The Boeing Company \\ Rocketdyne Propulsion and Power \\ Canoga Park, CA
}

\begin{abstract}
A three-dimensional flow solver has been developed for turbomachinery components utilizing real fluid properties. The code is applicable to both incompressible and compressible flow fields. In this study, the code has been applied to the analysis of inducer and impeller geometries representative of those used in rocket engine applications. The predicted results show good agreement with the available experimental data.
\end{abstract}

\section{NOMENCLATURE}

B2 - Non-dimensional channel width at exit

Qd - Design flow rate

P - Static pressure

P1 - Inlet static pressure

$\mathrm{X}$ - Axial location

$\phi \quad$ - Flow coefficient

\section{INTRODUCTION}

Turbomachines for propulsion applications operate on many different fluids and under a wide range of flow conditions. The working fluid may be air, liquid or gaseous hydrogen, liquid or gaseous oxygen, kerosene, etc. The flow may be incompressible, such as in the fuel pump in a liquid-fueled rocket engine, or supersonic, such as in the turbine that may drive the fuel pump.

Both compressible and incompressible flows are governed by the Navier-Stokes equations. However, in a nearly-incompressible flow there is a great disparity in wave speeds, since the speed of sound approaches infinity for a truly incompressible fluid. A compressible flow solver will encounter numerical stiffness if applied to a nearly-incompressible flow, and the algorithm will fail. Because of this, it is common practice to use one algorithm for incompressible flows and a different algorithm for compressible flows.

The General Equation Set (GES) method [1] has been developed to handle both compressible and incompressible flows. It can be used to solve the full, unsteady, three-dimensional Navier-Stokes equations, and with the introduction of a single input flag can reduce to the pseudocompressibility method commonly used to solve incompressible flows. When used in conjunction with a dual time step, it provides time accurate simulations. In addition, when the Navier-Stokes equations are cast in their general form, without applying the perfect gas relation, they can be

*Aerospace Engineer, Associate Fellow AIAA.

+ Senior Scientific Programmer, Senior Member AIAA.

\# Aerospace Engineer, Senior Member AIAA. 
solved for any working fluid for which properties are available. The GES technique in conjunction with the equations cast in this general form results in a general flow solver applicable to most conditions encountered in turbomachinery applications. A code has been written to solve three-dimensional, unsteady turbomachinery flows using the GES method.

Turbopump for upcoming space applications will need to operate over a wider range of flow conditions than current designs. Improving these designs requires detailed knowledge of the timeaveraged and transient flow fields for the operating range of interest. Previous works on this subject include Refs. 2-7. These references include works describing the application of numerical codes to both the analysis and design of rocket engine turbopump components. In this study, numerical simulations have been performed for a high-head impeller with a vane island diffuser operating in water, and an inducer operating in water, liquid hydrogen and air. The simulations were performed over a range of flow conditions. The predicted results have been compared with test data where available.

\section{NUMERICAL PROCEDURE}

The general code structure is based on a wellestablished compressible, 3-D, unsteady turbomachinery flow code [8]. It employs a system of overset $\mathrm{O}$-grids and $\mathrm{H}$-grids, with the values on the $\mathrm{O}-\mathrm{H}$ boundaries being updated each time step by bilinear interpolation from the adjacent grid. The inviscid fluxes are third-order spatially accurate and are calculated using Roe's scheme [9]. The viscous fluxes are calculated using second-order accurate central differences, and the code is second-order accurate in time. A modified Baldwin-Lomax turbulence model is used for turbulence closure [10].

The code contains two options for the determination of fluid properties. The first option is based on the equations of state, thermodynamic departure functions and corresponding state principles constructed by Oefelein [11]. The second option, used only for liquids, is based on splines generated from the NIST Tables [12], and is computationally more efficient. The code currently contains fluid property routines (both gaseous and liquid) for water, hydrogen, oxygen, nitrogen, RP-1, methane and carbon monoxide.

\section{Computational Grids}

The O-grids are body-fitted to the surfaces of the airfoils and generated using an elliptic equation solution procedure. They are used to properly resolve the viscous flow in the blade passages and to easily apply the algebraic turbulence model. The algebraically generated $\mathrm{H}$-grids are used to discretize the remainder of the flow field.

\section{Boundary Conditions}

For incompressible inlet flow the mass flow, total temperature, and the circumferential and radial flow angles are specified as a function of the radius. The static pressure is extrapolated from the interior of the computational domain.

For incompressible outflow two velocity components, static pressure, and the static temperature are extrapolated from the interior of the computational domain. The exit mass flow is specified.

Periodicity is enforced along the outer boundaries of the $\mathrm{H}$-grids in the circumferential direction.

At solid surfaces the relative velocity is set to zero, the normal derivative of the pressure is set to zero, and the surfaces are assumed to be adiabatic.

A detailed description of the code/algorithm development, as well as its application to several turbine and pump test cases, is presented in Refs. 13 and 14 .

\section{NUMERICAL TEST CASES}

Numerical simulations have been performed for two pump geometries:

1) A high-head impeller design which contains six main blades and six splitter blades. The impeller is followed by a vane-island diffuser containing 13 airfoils. The impeller rotates at 6322 RPM and was tested in water $(\mathrm{H} 2 \mathrm{O})$.

2) A low pressure fuel pump inducer containing four inducer blades. At design conditions the inducer rotates at approximately 16000 RPM. Simulations were performed using $\mathrm{H} 2 \mathrm{O}$, liquid hydrogen ( $\mathrm{LH} 2)$ and air to determine 
any differences in the predicted flow fields.

\section{High-Head Impeller and Diffuser}

The computational grid was generated using the actual airfoil counts of 6 main impeller blades, 6 splitter blades and 13 diffuser airfoils. The grid requires the solution of the full 360-degree annulus, and the computational grid was constructed using 4.8 million grid points. The full-annulus grid for the high-head impeller geometry is shown in Fig. 1.

The fluid properties for $\mathrm{H} 2 \mathrm{O}$ were obtained from the NIST data. The simulations were performed using preconditioning with two dual time steps for time accuracy. Simulations were performed for flow rates of $50 \%, 60 \%, 70 \%, 88 \%, 100 \%$ and $120 \%$ Qd.

Instantaneous and time-averaged nondimensional static pressure contours at midheight of the channel are shown for $100 \%$ Qd in Figs. 2 and 3. In Fig. 3 the static pressure was time-averaged over a complete impeller revolution. There are significant differences between the instantaneous and time-averaged solutions shown in the two figures. These differences are a direct result of the presence of the diffuser airfoils and its interaction with the passing impeller blades. The instantaneous contours in Fig. 2 show a significant amount of unsteadiness that extends from upstream of the impeller to downstream of the diffuser vanes. The presence of circumferential variations within the diffuser in Fig. 3 suggests the presence of unsteadiness at a frequency less than one per revolution, such as that associated with separated and/or vortical flows.

Figures 4 and 5 show comparisons of the predicted mid-passage radial and tangential velocity distributions across the impeller exit plane with experimental data [15]. The experimental data contain B2 locations less than zero and greater than one because the actual geometry has secondary flow paths (where flow is extracted and routed back to the inlet) located at the endwalls of impeller exit plane which were neglected in the current study. In general, there is good agreement between the predictions and the experimental data. Both data sets show increased flow towards the hub $(B 2=0)$ of the machine. This can be deduced by noting the shift in the peak radial velocity towards the hub. The decreased flow near the tip ( $B 2=1)$ is caused by a region of secondary flow.

Figure 6 shows predicted and experimental timeaveraged static pressure distributions on the diffuser shroud, beginning at the diffuser throat and moving downstream. The current simulation over-predicts the static pressure near the throat of the diffuser, but shows good agreement with the experimental data downstream of the throat. The differences near the throat are likely caused by neglecting the secondary flow path cavities at the impeller exit.

Figures 7 and 8 contain the predicted and experimental static head coefficients for the impeller and diffuser at various flow coefficients [15]. Both the data and the predictions show the impeller static head coefficient remains relatively constant over the range of flow coefficients investigated in this study (see Fig. 7). The predicted head coefficients for the diffuser show the rapid decrease in head coefficient as the flow coefficient is reduced and the diffuser stalls. The predicted diffuser stall occurs at a higher flow coefficient than in the experiment, but additional simulations are needed between flow coefficients of 0.10 and 0.13 to resolve the shape of the stall region shown in Fig. 8. Both sets of data show a decrease in the head coefficient at flow coefficients greater than the design value of 0.144 . Overall, there is good agreement between the predicted results and the experimental data.

\section{Low Pressure Fuel Pump Inducer}

The computational grid was generated including all four inducer blades and contains 2.8 million grid points. The surface grid for the inducer geometry is shown in Fig. 9. Unless otherwise specified, all the results presented below are for the inducer operating in LH2 at a flow coefficient of $\phi=0.70$ (approximately $65 \%$ rated power level).

The fluid properties for $\mathrm{LH} 2$ and $\mathrm{H} 2 \mathrm{O}$ were obtained the NIST data, while the air was assumed to be an ideal gas.

Time-averaged non-dimensional static pressure contours in the inducer are presented in Fig. 10. The low pressure region near the leading edge tip is characteristic of most inducers, and is coincident with the location where cavitation is frequently observed in experiments. The contours also suggest a nearly linear pressure rise 
through the inducer. Figure 11 shows instantaneous non-dimensional pressure contours on a cross-section of the inducer. This figure further elucidates the pressure increase through the inducer, and shows the effects of the inducer on the upstream pressure field.

Time-averaged blade surface pressure distributions at $10 \%, 50 \%$ and $90 \%$ of the blade height are presented in Fig. 12. The overall blade loading is observed to increase with increasing span. The leading edge portion of the inducer is unloaded in the hub region, and is heavily loaded in the tip region. The heavy loading near the leading edge tip coincides with the low pressure region shown in Fig. 10.

Figure 13 shows unsteady pressure traces at several locations along the duct outer wall upstream of the inducer. The inducer tip leading edge is located at $x=0.0$ inches, so the locations shown in Fig. 12 correspond to 1.0, 2.0, 3.0 and 4.0 inches upstream of the inducer. Note, the inducer tip radius is approximately 6 inches. The amplitude of the unsteadiness 1 inch upstream of the inducer is large, on the order of the free stream pressure. This unsteadiness is due in part to the large pressure difference across the leading edge tip region (tip clearance was not modeled in this study), which generates backflow from the inducer. The potential field of the inducer also contributes to the unsteadiness upstream. The unsteadiness decays exponentially upstream of the inducer, such that 4 inches upstream of the inducer leading edge little unsteadiness is observed. Figure 14 contains the Fourier decompositions of the traces shown in Fig. 13. The primary frequency for the unsteadiness is the inducer blade passing frequency $(4 \mathrm{~N})$, with unsteadiness also observed at twice the blade passing frequency $(8 \mathrm{~N})$ just upstream of the inducer.

The decay of the amplitude of the unsteady pressure upstream of the inducer leading edge is compared with the results of an analytical wave model [16] and data from a hot fire test performed at Stennis Space Center [17] in Fig. 15. The analytical wave model requires the unsteady pressure amplitude just upstream of the inducer as input. This value was obtained from the CFD solution. The test data and wave model are nearly coincident, while the predicted wave decay shows good agreement with both the wave model and experimental data. The more rapid wave decay in the simulations may indicate the need for increased grid density in either (or both) the axial and radial directions.

Figure 16 contains the static head coefficients from simulations in air, $\mathrm{H} 2 \mathrm{O}$ and $\mathrm{LH} 2$, along with experimental data obtained from recent water flow tests [18]. The predicted value in LH2 at a flow coefficient of $\phi=0.70$ exhibits excellent agreement with the data, while the $\mathrm{LH} 2$ resuit at $\phi=1.00$ is slighter larger than shown in the experimental data. The predicted results for air and $\mathrm{H} 2 \mathrm{O}$ show close agreement with the $\mathrm{LH} 2$ results, suggesting that water flow tests are adequate for determining the overall performance of pumps operating in cryogenic fluids.

Figure 17 shows a cross-sectional view of nondimensional axial velocity contours, highlighting the backflow upstream of the inducer. The back flow extends more than 13 inches upstream of the inducer leading edge, which qualitatively agrees with video of tufts placed upstream of the inducer in the experiments.

\section{CONCLUSIONS}

A real-fluid turbomachinery analysis has been applied to impeller and inducer geometries operating in $\mathrm{H} 2 \mathrm{O}, \mathrm{LH} 2$ and air. The predicted results show good agreement with the available experimental data, and have yielded insights into the unsteady flow mechanisms in these turbomachines.

Current work includes the debugging and validation of a cavitation model implemented into the code, and improving the turbulence modeling.

\section{ACKNOWLEDGEMENTS}

The authors would like to acknowledge the use of the supercomputer facilities at NASA Ames Research Center, and especially thank $\mathrm{Mr}$. Chuck Niggley for his assistance. The authors would also like to thank Mr. Morgan Williams of Rocketdyne for his many helpful discussions, and for providing the reference with the linear wave model.

\section{REFERENCES}

1. Venkateswaran, S. and Merkle, C. L., "Analysis of Preconditioning Methods for the Euler and Navier-Stokes Equations, Von 
Karman Institute Lecture Series, March 8 12, 1999.

2. Graf, E. 1993. "Analysis of Centrifugal Impeller BEP and Recirculating Flows: Comparison of Quasi-3D and Navier-Stokes Solutions," ASME Fluids Engineering Conference Second Pumping Machinery Symposium, June 1993, Washington D.C., Vol. 154, pp. 235-245.

3. Brozowski, L. A., Ferguson, T. V., Lee, G. A., Prueger, G. H., and Rojas, L., 1993. "Laser Velocimeter Measurements of an Impeller Flow Field,".ASME Fluids Engineering Conference Second Pumping Machinery Symposium, June 1993, Washington D.C., vol. 154, pp. 187-196.

4. Chen, W-C., Prueger, G. H., Chan, D. C. and Eastland, A. H, 1992. "On the Use of a Three-Dimensional Navier-Stokes Solver for Rocket Engine Pump Impeller Design," AIAA/SAE/ASEE, 28 $8^{\text {th }}$ Joint Propulsion Conference and Exhibit, Nashville, July 6-8, 1992.

5. Garcia, R., Jackson, E. D., and Schutzenhofer, L. A. 1992. "A Summary of the Activities of the NASA/MSFC Pump Stage Technology Team," $4^{\text {th }}$ ISROMAC, Hawaii, April 1992.

6. Guinzburg, A., Williams, M., Brozowski, L., and Chen, W.-C. "Effect of Inlet Conditions on the Numerical Results of a Centrifugal Pump Compared with Experimental Conditions," AIAA Paper 98-3998, 1998.

7. Guinzburg, A. and Williams, M., "Advanced Deep Throttling Pump Diffuser Concept Development," JANNAF $27^{\text {th }}$ Airbreathing Subcommittee Meeting, Colorado Springs, CO, Dec. 1-5, 2003.

8. Dorney, D. J., and Schwab, J. R., "Unsteady Numerical Simulations of Radial Temperature Profile Redistribution in a Single-Stage Turbine," ASME Journal of Turbomachinery, Vol. 118, No. 4, October 1996, pp. 783-791.

9. Roe, P. L., "Approximate Riemann Solvers, Parameter Vectors, and Difference Schemes," Journal of Computational Physics, Vol. 43, 1981, pp. 357-372.

10. Baldwin, B. S., and Lomax, H., 'Thin Layer Approximation and Algebraic Model for Separated Turbulent Flow," AIAA Paper 78-257, Huntsville, AL, January, 1978.

11. Oefelein, J. C., Sandia Corporation, Livermore, CA, Private Communication, December, 2002.

12. http://webbook. nist.gov/chemistry/fluid
13. Sondak, D. L. and Dorney, D. J., "General Equation Set Solver for Compressible and Incompressible Turbomachinery Flows," AIAA 2003-4420, 39th AIAA/ASME/SAE/ASEE Joint Propulsion Conference and Exhibit, Huntsville, AL, July 20-23, 2003.

14. Merkle, C. L., Venkateswaran, S., Dorney, D. I., and Sondak, D. L., “A Generalized Fluid Formulation for Turbomachinery Computations," AIAA 2003-3999, 33rd AIAA Fluid Dynamics Conference and Exhibit, Orlando, FL, June 23-26, 2003

15. Brozowski, L., “Advanced Pump Diffuser Final Report”, Rockwell International, Rocketdyne Division, Report RSS-8895-42, August, 1996.

16. Abd-El-Malak, M. B., Hanna, S. N., "'Sound Attenutation in a Circular Duct of a Viscous Medium in the Absence of Mean Flow," Nonlinear Mathematical Physics, Vol. 4, No. 1-2, 1997, pp. 231-240.

17. Zoladz, Thomas, NASA Marshall Space Flight Center, MSFC, AL, Private Communication, August, 2004.

18. Skelley, S. "SSME Low Pressure Fuel Pump Water Flow Test (P2397)", Preliminary Data Review \#2, NASA Marshall Space Flight Center, August, 19, 2004. 


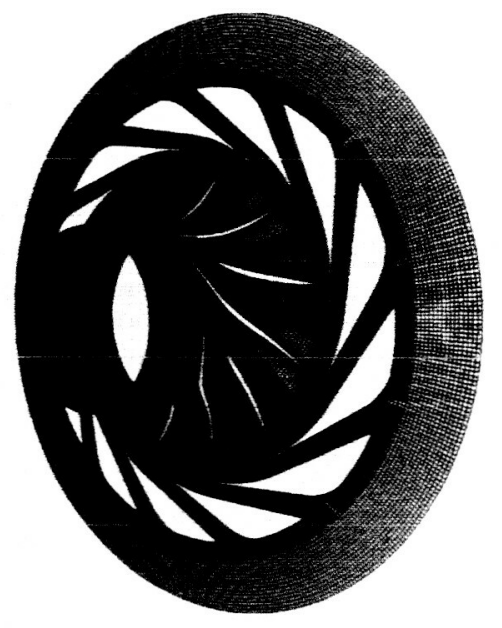

Figure 1. Computational grid for the high-head impeller/diffuser.

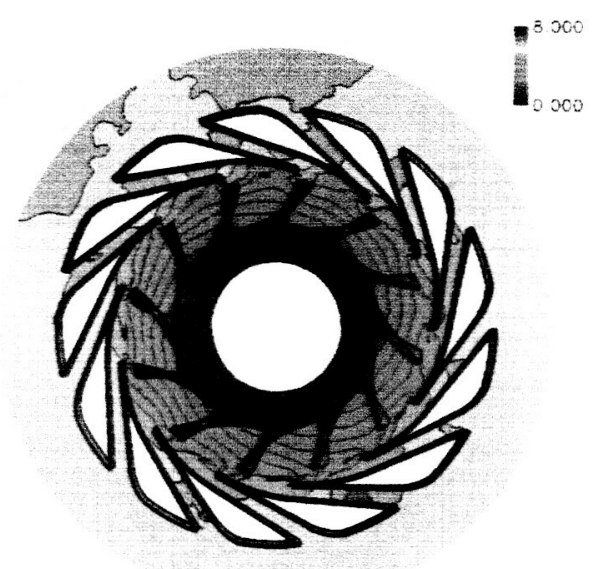

Figure 2. Instantaneous static pressure contours.

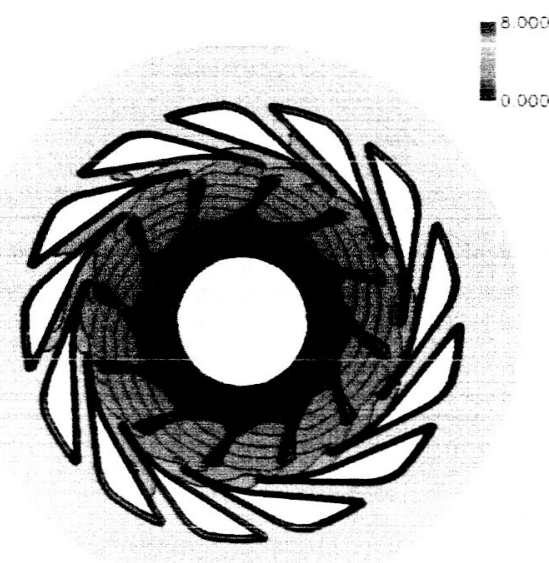

Figure 3. Time-averaged static pressure contours.

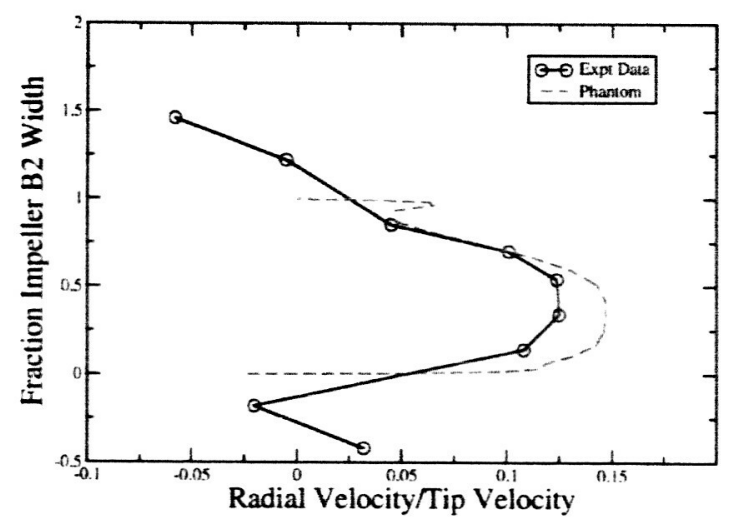

Figure 4 . Radial velocity at the impeller exit. 


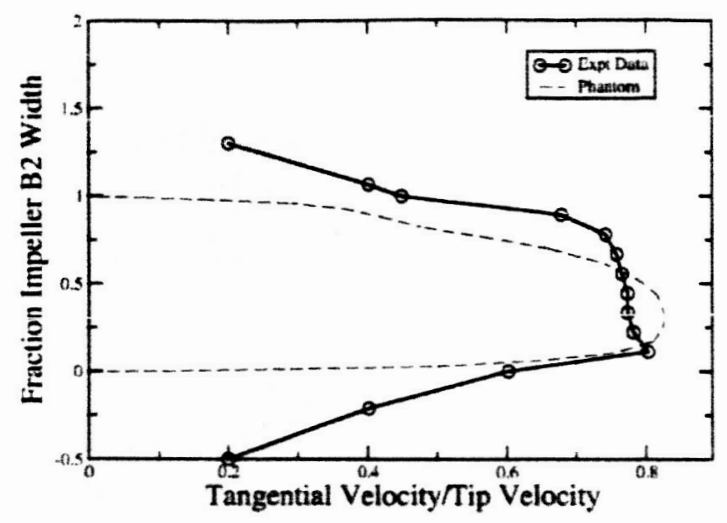

Figure 5. Tangential velocity at the impeller exit.

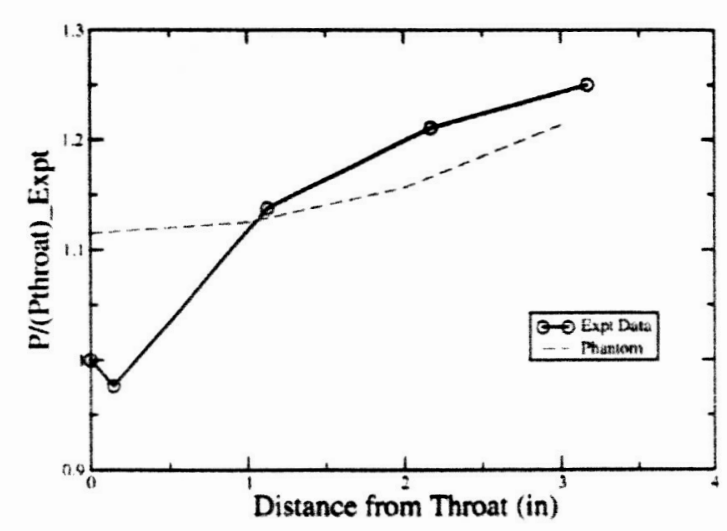

Figure 6. Static pressure on the diffuser shroud.

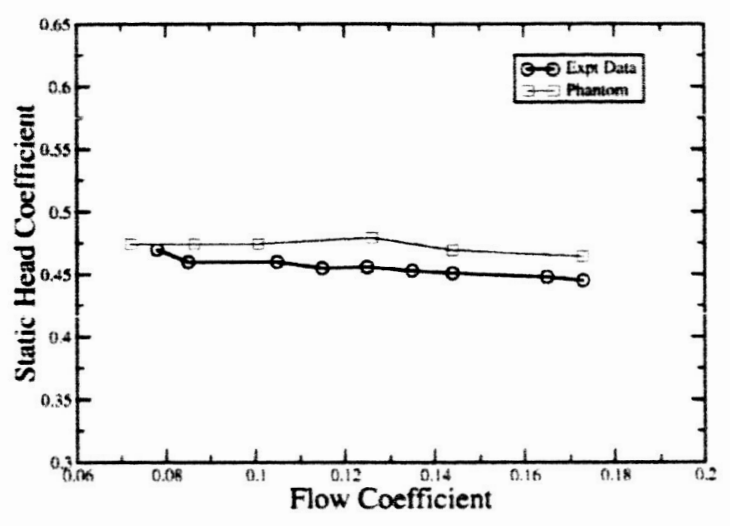

Figure 7. Impeller static head coefficient.

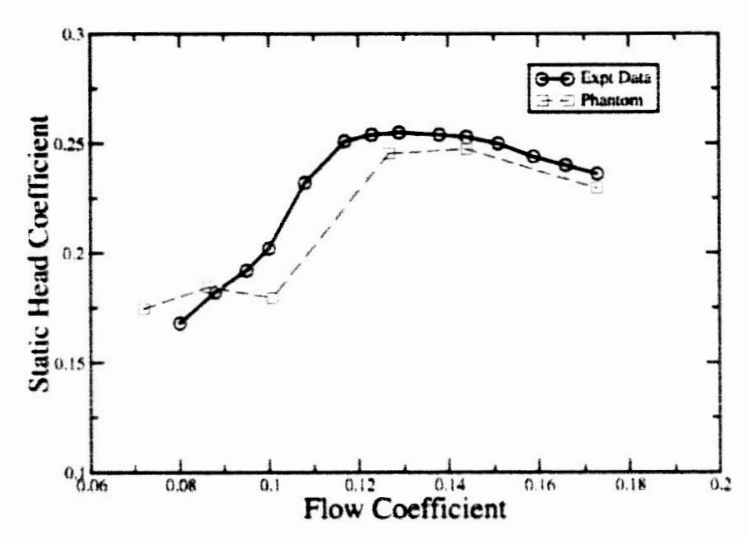

Figure 8. Diffuser static head coefficient. 


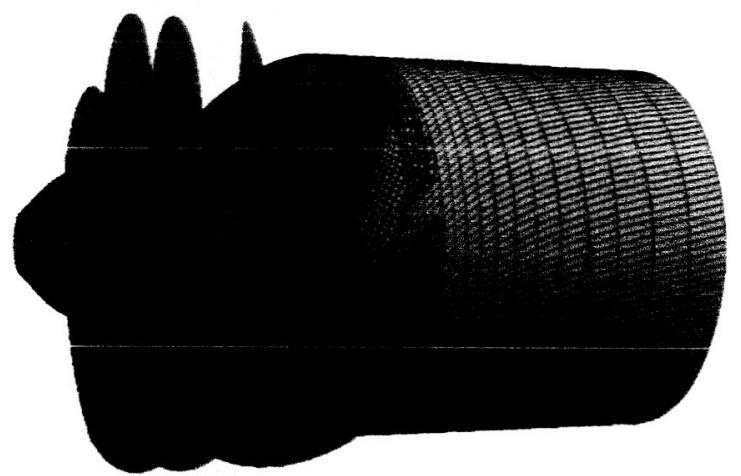

Figure 9. Computational grid for the inducer.

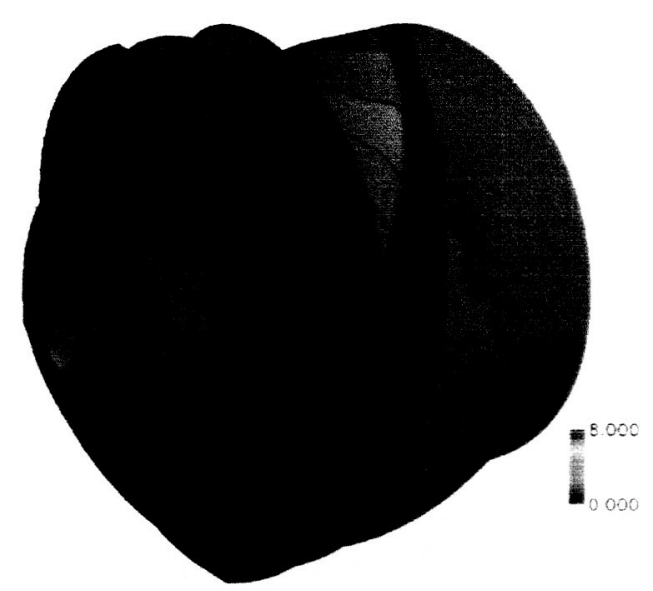

Figure 10. Time-averaged static pressure contours in the inducer.

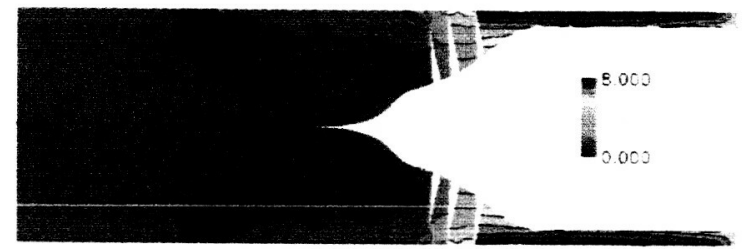

Figure 11. Cross-section view of the static pressure in the inducer.

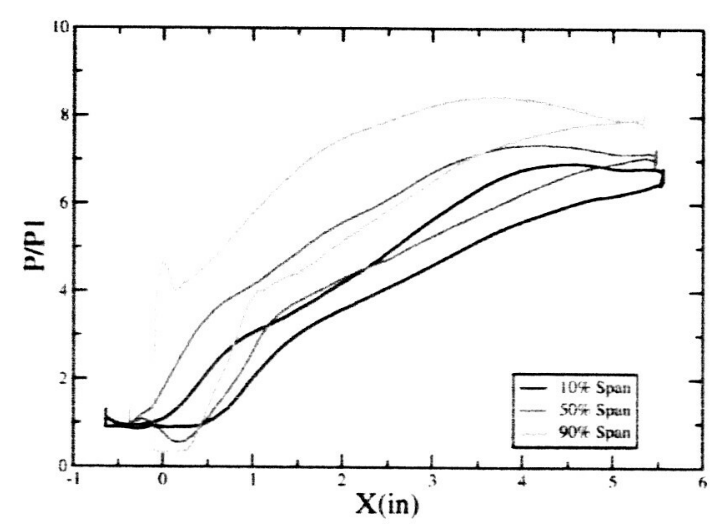

Figure 12. Time-averaged surface pressure distributions on the inducer. 


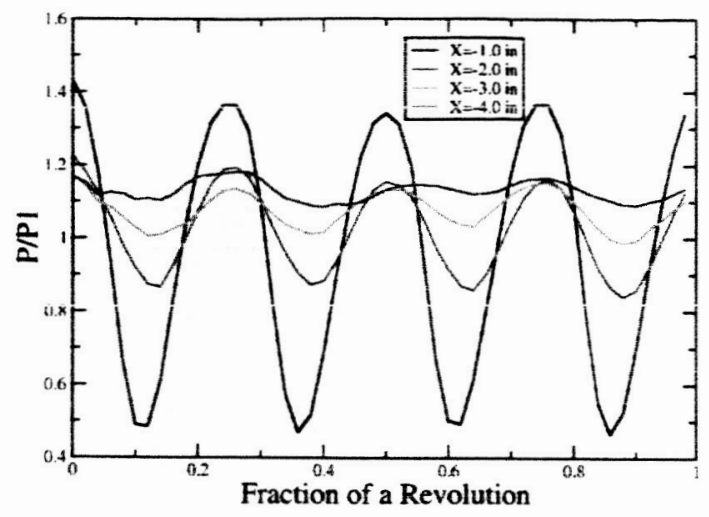

Figure 13. Unsteady pressure traces along the outer duct wall, upstream of the inducer.

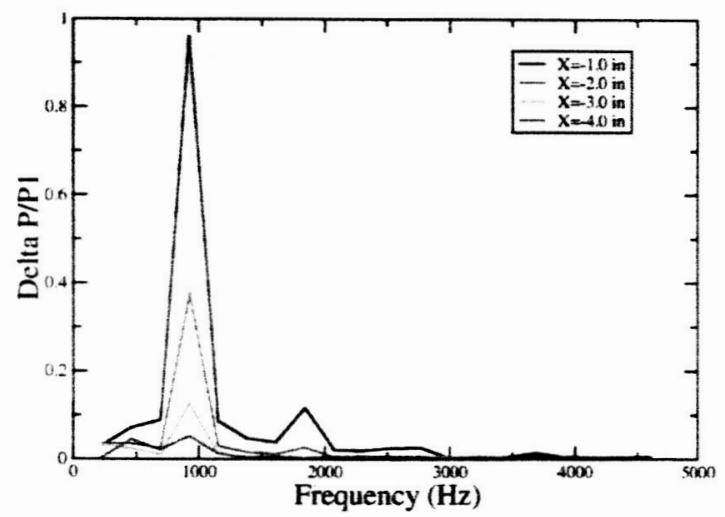

Figure 14. Decomposition of the unsteady pressure on the duct wall, upstream of the inducer.

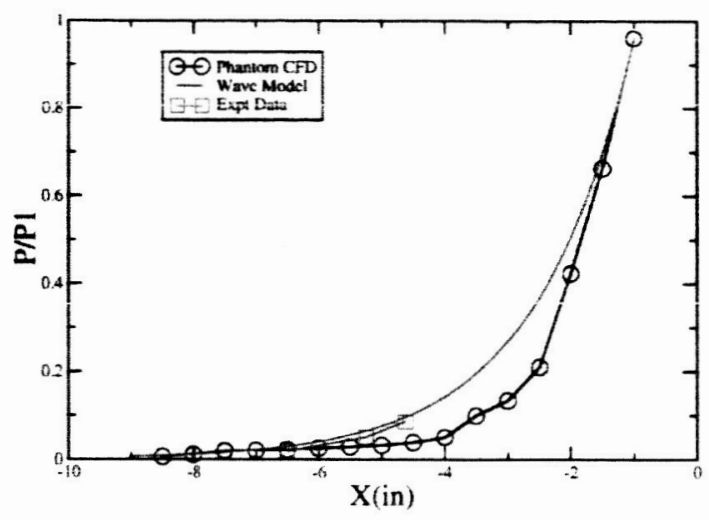

Figure 15. Unsteady pressure amplitude decay along the outer duct wall - predicted, analytical and experimental.

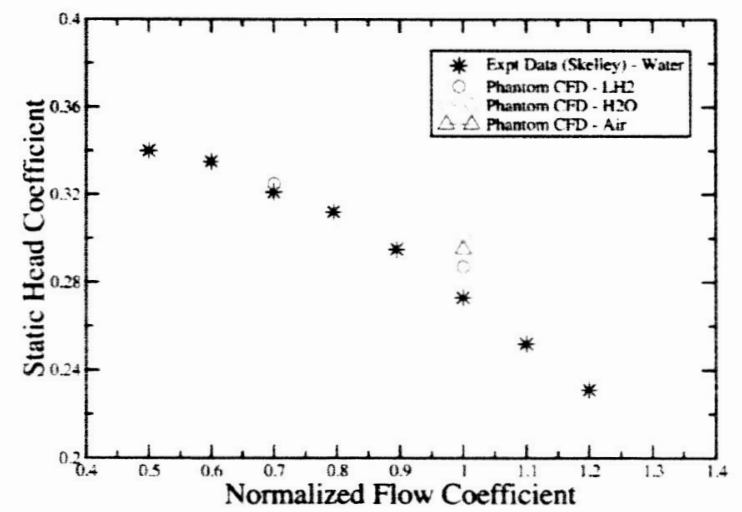

Figure 16. Predicted and experimental static head coefficient data for the inducer. 


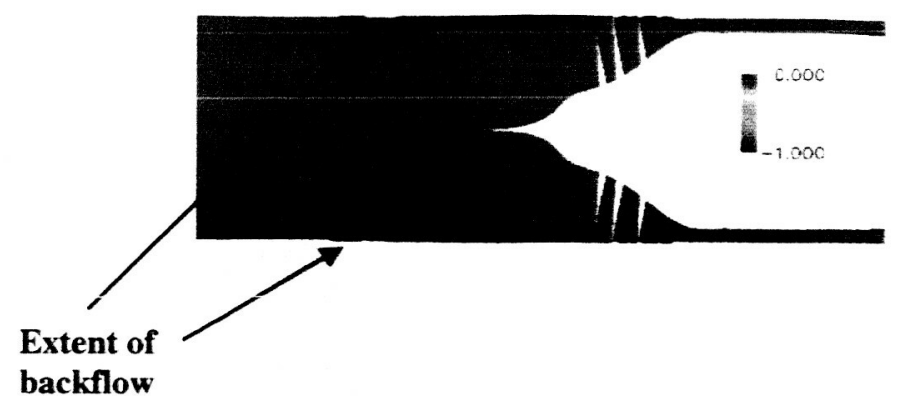

Figure 17. Non-dimensional axial velocity contours showing the extent of the backflow. 